\title{
Are Changes in Beliefs About Rumination and in Emotion Regulation Skills Mediators of the Effects of Internet-Delivered Cognitive-Behavioral Therapy for Depression and Anxiety? Results from a Randomized Controlled Trial
}

\author{
Angel Enrique ${ }^{1,2}$ - Nora Eilert ${ }^{1,2} \cdot$ Rebecca Wogan $^{2} \cdot$ Caroline Earley $^{1,2} \cdot$ Daniel Duffy $^{1,2} \cdot$ Jorge Palacios $^{1,2}$. \\ Ladislav Timulak ${ }^{1} \cdot$ Derek Richards $^{1,2}$
}

Accepted: 18 December 2020 / Published online: 18 January 2021

(c) The Author(s) 2021

\begin{abstract}
Background Internet-delivered cognitive behavior therapy (iCBT) is effective in treating anxiety and depression. Research on how these interventions operate is scarce. This study explored whether emotion regulation skills and positive beliefs about rumination were affected by iCBT and if these constructs mediated changes in depression and anxiety.

Methods This is a secondary analysis of a pragmatic randomized waitlist-controlled trial testing the effectiveness of supported iCBT. Adults with at least mild symptoms of depression or anxiety were included. Depression (PHQ-9), anxiety (GAD-7), positive beliefs about rumination (PBRS-A) and two emotion regulation skills: cognitive reappraisal (ERQ-A) and expressive suppression (ERQ-S), were measured at baseline and 8-weeks post-treatment.

Results The analyses included 358 participants, $71 \%$ were female. Median age was 29 . Linear mixed models showed statistically significant differences along ERQ-A in favor of the iCBT group $(b=1.83, S E=0.82, p=.026)$. Mediation analyses showed reductions in depression $(b=0.31, S E=0.15, p=0.043)$ and anxiety symptoms $(b=0.27, S E=0.14, p=0.057)$ were partially mediated by gains in ERQ-A. No effects were observed for PBRS-A and ERQ-S.

Conclusions These results align with findings from face-to-face therapy and add to the scarce literature on mediators of effects of iCBT, contributing to the understanding of how these interventions operate. Since mediator and outcome variables were measured at the same time, partial mediation results should be interpreted with caution since the study design did not account for temporality and therefore causality effects cannot be confirmed.
\end{abstract}

Trial Registration ISRCTN91967124.

Keywords Mediators of change $\cdot$ Anxiety $\cdot$ Depression $\cdot$ CBT $\cdot$ Internet-delivered treatment

\section{Introduction}

Common mental health disorders (CMD), such as depression and anxiety, are highly prevalent and global (James et al. 2018). These disorders are associated with pervasive and debilitating effects including impairment in daily

Angel Enrique

enriquea@tcd.ie

1 E-Mental Health Research Group, School of Psychology, Aras an Phiarsaig Trinity College, University of Dublin, Dublin, Ireland

2 Clinical Research \& Innovation, SilverCloud Health, One Stephen Street Upper, Dublin 8, Ireland functioning and low quality of life (Salomonsson et al. 2019), physical disability (Brenes et al. 2008), and elevated risk of suicide (Grek 2007). In addition to high individual prevalence rates, these disorders display high levels of comorbidity, with $40-80 \%$ of people diagnosed with major depression also meeting diagnostic criteria for an anxiety disorder (Kessler et al. 2007; Wu and Fang 2014). Various psychological therapies have shown effectiveness in treating symptoms of anxiety and depression (Cuijpers et al. 2008). However, there is still a global treatment gap between the prevalence of CMD and individuals receiving adequate treatment for depression and anxiety (Alonso et al. 2018; Thornicroft et al. 2017). This gap is due to many different reasons, such as costs, shortages of adequately qualified 
practitioners in healthcare services, long waiting times, stigma and incompatibility of traditional face-to-face therapy with patients' lifestyle (Bennett-Levy et al. 2010; Alonso et al. 2018).

Internet-delivered psychological interventions are proposed as a solution to increase access to evidence-based psychological treatments as it requires less therapist involvement and can be more flexibly delivered (Dear et al. 2015). In these interventions, clients complete the structured therapy through an online platform but can still be supported by clinical staff via phone or email (Andersson and Cuijpers 2009). Internet-delivered Cognitive-Behavioral Therapy (iCBT) has received a lot of empirical attention and several meta-analyses have shown that these interventions are effective in treating depression and anxiety compared to control groups (Andrews et al. 2018; Wright et al. 2019).

These interventions have shown benefits in different populations and settings; however, it is still unclear what the underlying mechanisms are that mediate the success of these interventions. Understanding the mechanisms of change in psychotherapy allows us to enhance optimal treatment outcomes and reduce detrimental effects (Kazdin 2007). These mechanisms can be studied by means of mediation analyses that examine the strength of association between the treatment, a potential mediator, and the symptom outcomes where the mediator may function in a dose-response relationship (Holmes et al. 2018). One may assume that the underlying specific mechanisms contributing to change in iCBT are the same as those in traditional CBT, as it is explicitly designed as a digitalized version of face-to-face therapy. However, given the differences in design and implementation, more studies are required to confirm whether the active mechanisms that are apparent in traditional CBT are also relevant to iCBT.

A review conducted by Mogoașe et al. (2017) explored the mechanisms of change that have been found in iCBT determining that the research on this topic is still in its infancy. Evidence to date has found that dysfunctional attitudes, worry, negative problem orientation, and cognitive skill acquisition mediate the effects of iCBT on outcomes (Forand et al. 2018). Specifically, positive beliefs surrounding the functionality of repetitive negative thinking were found to mediate the effects of iCBT on comorbid anxiety and depression (Newby et al. 2014). Furthermore, treatments specifically targeting rumination, such as rumination-focused iCBT, were found to be effective at preventing anxiety and depressive symptoms in high-risk individuals (Topper et al. 2017; Cook et al. 2019). It has been proposed that positive beliefs surrounding rumination act as a contributing factor in the maintenance of distress of individuals with generalized anxiety and depressive disorders (McEvoy et al. 2015) and changes to these may form the basis of improvements arising from CBT interventions (Kertz et al. 2015).

Emotion regulation is another construct that has been proposed as a transdiagnostic underlying mechanism in treatments for depression and anxiety (Aldao et al. 2010; Sloan et al. 2017). A meta-analytic review found that maladaptive strategies such as emotional avoidance and suppression, were positively correlated with pre-treatment symptoms of anxiety and depression, while adaptive strategies, such as reappraisal, were found to be negatively correlated (Aldao et al. 2010). A study of a transdiagnostic internet intervention for patients following hospital discharge from inpatient psychotherapy found that emotion regulation skills were improved compared to a treatment as usual group and these differences were maintained at 12-months follow up (Ebert et al. 2013). Although these findings suggest that emotion regulation skills may be impacted by iCBT interventions, more research is needed to understand the role that this construct may play and whether it can be considered a mechanism of change.

Given the dearth of research to date, opportunities to examine mechanisms of change are welcome as it is clear that more empirical investigation is needed regarding mediators of these mechanisms and their influence on improving the therapeutic outcomes. Furthermore, there is much diversity in iCBT protocols and further investigation of additional cognitive factors will aid in identifying the essential components of $\mathrm{iCBT}$ in order to produce real clinical change (Mogoașe et al. 2017). This paper attempts to address these issues, specifically, we examine (i) whether the iCBT intervention is effective at improving emotion regulation skills and positive beliefs about rumination and (ii) whether changes in positive beliefs about rumination and emotion regulation skills mediate the effects of the iCBT intervention on depression and anxiety outcomes.

\section{Methods}

\section{Trial Design}

This research was conducted as a secondary analysis nested within a larger RCT examining the effectiveness and costeffectiveness of supported iCBT for treating anxiety and depression (Richards et al. 2018). Three-hundred and sixtyone participants $(\mathrm{n}=361)$ were randomized to the treatment $(n=241)$ or the waiting list $(n=120)$ groups in a 2:1 ratio to reduce the number of participants waiting for treatment. The primary outcome measures of the RCT were depression and anxiety, assessed using the Patient Health Questionnaire-9 (PHQ-9) (Kroenke et al. 2001) and the Generalized Anxiety Disorder-7 (GAD-7) (Spitzer et al. 2006) respectively. Results showed reductions in depression and anxiety 
symptoms at post-treatment compared to the control group and a further symptom improvement over 12 months follow up (Richards et al. 2020). Emotional regulation skills and positive beliefs about rumination were included as secondary measures and administered at baseline and 8-week research timepoints for all participants. The trial protocol was approved by the NHS England Research Ethics Committee [REC Reference: 17/NW/0311].

\section{Study Setting}

The current study was conducted at step 2 of the Improving Access to Psychological Therapies (IAPT) in the UK. Step 2 offers low-intensity treatments for mild to moderate presentations of depression and anxiety, such as guided self-help, internet-delivered CBT or group psychoeducation, supported by specially trained Psychological Wellbeing Practitioners (PWP). This service aims to provide an effective and widely accessible stepped-care treatment model for anxiety or depression. Clients are referred through a GP, allied services or through self-referral. For this study, participants provided written consent and are unidentifiable from the data.

\section{Eligibility Criteria}

All adult users at step 2 services were eligible to participate if they received a score $\geq 9$ on the PHQ-9 and/or $\geq 8$ on the GAD-7 and if they were suitable for iCBT. Suitability and willingness to engage with internet-delivered treatment were assessed by the PWPs over the telephone, determined by participants having access to the internet, low to moderate levels of anxiety or depression, and no suicidal or self-harm risk. The exclusion criteria were suicidal intent/ideation marked by a score $>2$ on the PHQ-9 question 9, diagnosis of a psychotic illness, currently in psychological treatment for depression or anxiety, and a history of substance abuse.

\section{Assessments}

\section{Patient Health Questionnaire-9 Item (PHQ-9)}

The PHQ-9 (Kroenke et al. 2001) is a self-report questionnaire used to measure depression symptoms over the previous 2 weeks, regularly used in healthcare settings. The scoring ranges from 0 to 27 , where larger scores reflect greater severity and frequency of depressive symptoms. The scale uses a 4-point Likert scale to describe symptom presence in which $0=$ 'Not at all' and $3=$ 'Nearly every day'. The PHQ-9 has been found to discriminate well between depressed and non-depressed individuals using the cut-off total score $\geq 10$, with good sensitivity $(88.0 \%)$, specificity $(88.0 \%)$ and reliability $(89,39)$ (Kroenke et al. 2001).

\section{Generalized Anxiety Disorder-7 Item (GAD-7)}

The GAD-7 (Spitzer et al. 2006) is a self-report questionnaire measuring the frequency of anxiety symptoms over the previous 2 weeks. The scale has good internal consistency $(\alpha=0.92)$ and good convergent validity with other anxiety scales (Spitzer et al. 2006). Scores range from 0 to 21 , where higher scores indicate greater severity of anxiety symptoms. It is assessed using a 4-point Likert scale ranging from $0=$ 'Not at all' and $3=$ 'Nearly every day'. The GAD-7 is often used in large-scale studies to measure change in anxiety symptomatology, with a cut-off score of 8 . It has shown good internal consistency (Cronbach's $\alpha=83$; Rodebaugh et al. 2008), and good test-retest reliability ( $k=64$ over 2 weeks; Newman et al. 2002).

\section{Positive Beliefs About Rumination Scale-Adapted Version (PBRS-A)}

The PBRS-A (Watkins and Moulds 2005) is an adapted version of the Positive Beliefs about Rumination Scale (Papageorgiou and Wells 2001) which measures how helpful a person regards rumination. The adapted version removed explicit references to depression and rumination to reduce criterion contamination. For example, "I need to ruminate about my problems to find answers to my depression" was changed to "I need to think about things to find answers to how I feel". The PBRS-A is a 9-item scale which measures positive metacognitive beliefs about the functions of rumination. The scores on the scale range from 9 to 36, where participants answer each item using a 4-point scale where $1=$ 'Do not agree' and $4=$ 'Agree very much'. The measure has been shown to have good internal reliability with $\alpha=0.89$ (Watkins and Moulds 2005).

\section{Emotion Regulation Questionnaire (ERQ)}

The ERQ is a self-report measure consisting of 10 items assessing how emotions are controlled or regulated. Responses are scored on a Likert scale, within a range of 1-7 ('strongly disagree' to 'strongly agree') measuring the extent to which two strategies of regulating emotions are engaged in, cognitive reappraisal and expressive suppression (6-item reappraisal subscale, ERQ-A; 4-item suppression subscale, ERQ-S) (Gross and John 2003). Cognitive reappraisal refers to the ability to change how someone thinks about potentially emotion-eliciting events, which occurs early in emotional processing and correlates with positive wellbeing and interpersonal functioning (Ioannidis and Siegling 2015). Expressive suppression is a reactive response inhibiting expression of a fully-developed emotional response and is associated with reduced well-being and social inauthenticity (Ioannidis and Siegling 2015). Higher scores on the 
subscales reflect greater engagement in that strategy. Internal reliability is considerable, where Cronbach's alpha is 0.73 for suppression and 0.79 for reappraisal (Gross and John 2003).

\section{Mini International Neuropsychiatric Interview 7.0.2 (M.I.N.I.)}

This diagnostic interview was developed to assess disorders based on the Diagnostic and Statistical Manual of Mental Disorders (DSM-V) and International Classification of Diseases (ICD-10) criteria (Sheehan et al. 1997). The interview has been well validated and the administration of the interview by telephone has been demonstrated to have good validity. For this specific study, the interview included the following modules: Module A (Major Depressive Episode), Module D (Panic Disorder), Module F (Social Anxiety Disorder), and Module N (Generalized Anxiety Disorder), to determine current depression and anxiety conditions, including specific anxiety presentations.

\section{Interventions}

The online interventions used were 'Space from Depression','Space from Anxiety', and 'Space from Depression and Anxiety' created by SilverCloud Health, each comprised of 7-core modules, delivered on a Web 2.0 platform using media-rich interactive content. They are comprised of core CBT content not delivered in a set linear pattern, with customizations for depression, anxiety, and comorbid presentations, described in detail elsewhere (Richards et al. 2018). An overview of the modules is outlined in Table 1. The program 'Space from Depression and Anxiety' consists of modules from the depression program with an integrated worry component. Depending on the needs of the patient, there are additional modules that can be unlocked by their supporter, for example, one addressing the identification and confrontation of core beliefs. The interventions have been shown to be effective for the treatment of depression and anxiety in previous studies (Richards et al. 2015, 2016) and follow the guidelines of the National Institute for Health and Care Excellence (NICE 2009, 2011).

Each participant was supported by a Psychological Wellbeing Practitioner (PWP), who are psychology graduates specially trained in low-intensity CBT delivery within IAPT. PWPs received training in the iCBT program, including the content of the program and the manner of giving feedback to client input. All participants in the intervention group received a welcoming and informational message from their PWP upon initial sign-in, encouraging them to engage with the intervention. Throughout the 8 weeks of treatment, PWPs monitored client progress every 7-10 days and provided approximately $10-15$ min of post-session feedback to participants individually.

\section{Procedure}

Details of the procedure are outlined in the primary study (see Richards et al. 2020). Participants' beliefs about rumination were measured by their scores on the PBRS-A and their emotional regulation skills using the ERQ, at baseline and 8 weeks later (i.e. post-treatment). The waiting-list control (WL) group also completed the same measures at these time points but did not receive any therapy during the 8 -week intervention period. On completion of the 8-week period, participants in the WL group were offered access to treatment. Participants received financial incentives upon completion of these specific research measures, which are beyond the routine measures [PHQ-9, GAD-7] collected during treatment.

\section{Statistical Analyses}

Independent t-test and chi-squared tests were used to assess differences between intervention-arms along baseline demographic, outcome and mediator variables. Three participants who did not complete PBRS-A and ERQ measures at baseline or 8-weeks were excluded from the analysis, leaving a total of 358 participants included in the analyses. Missing data across variables was quantified and missing data mechanisms were evaluated through Little's 'missing completely at random' (MCAR) test. Random intercept linear mixed models with restricted maximum likelihood estimation were employed to explore whether intervention-arm assignment affected participants use of emotion regulation strategies (as measured by the ERQ) or their positive beliefs about rumination (as measured by the PBRS-A) at 8-weeks from baseline. Missing data, occurring at baseline and 8-weeks, was handled through multiple imputation via multilevel-joint modelling across these models (Grund et al. 2016; Huque et al. 2018). Bonferroni adjusted paired comparisons based on estimated marginal means were conducted to assess follow-up differences between arms and time-points.

Meditation analyses utilized a structural equational modeling approach to evaluate indirect effects of the three hypothesized mediators on clinical outcomes, extending Baron and Kenny's (1986) basic mediation model. Two separate parallel multiple mediation models were built to account for changes in levels of depression and anxiety, so changes in PHQ-9 and GAD-7 scores from baseline to 8 -weeks, respectively. Apart from a direct effect of intervention-arm on clinical outcome, the models estimated three separate indirect effects for ERQ-A, ERQ-S and PBRS-A, which were the product of the effect of intervention-arm on the mediator and the effect of the mediator 


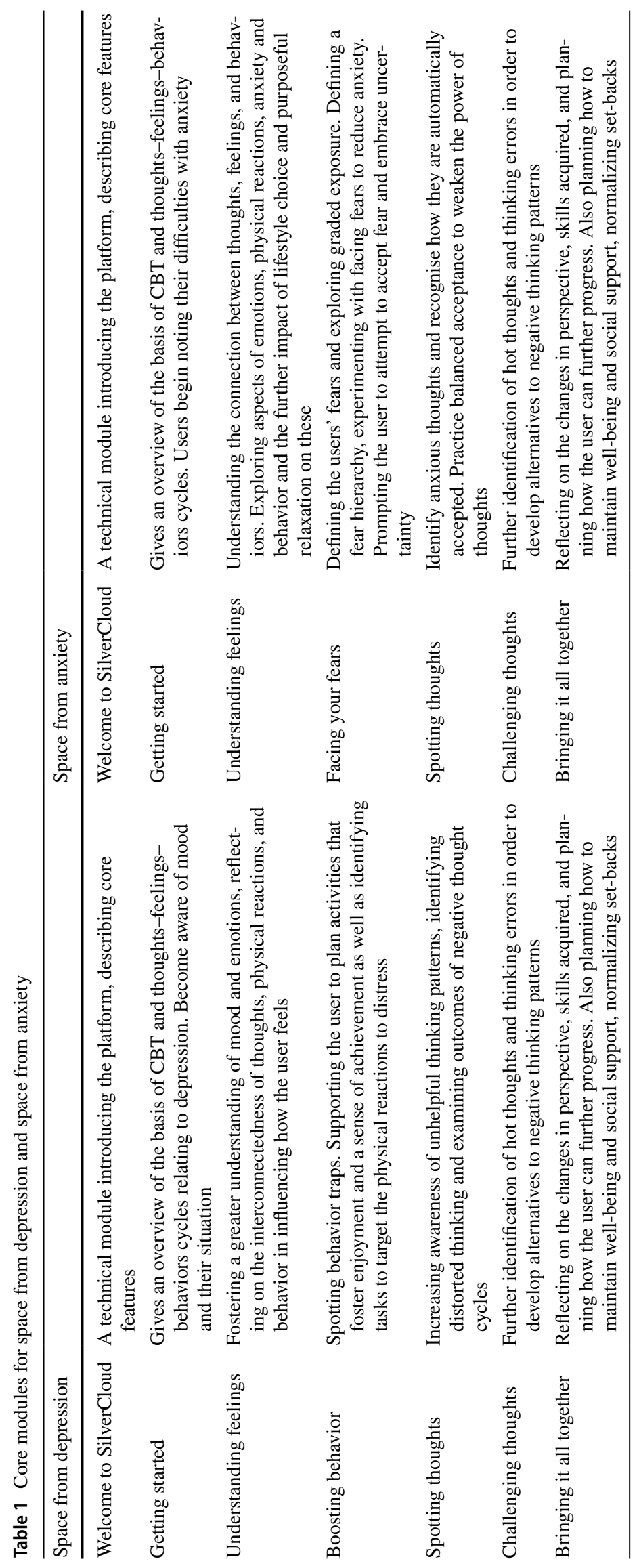


on clinical outcome. Mediation was assumed if both these paths as well as its product, the indirect effect, were significant and the direct effect was smaller in size than the total effect, the sum of the direct and indirect effect. Missing data was handled through Full Information Maximum Likelihood procedures, robust standard errors in line with Yuan and Bentler (2000) were implemented and various fit indices for the models were obtained to ensure good model fit. Where significant mediation effects were discovered, post hoc confirmatory factor analyses were conducted to determine the degree to which mediator and outcome constructs overlapped. To this end, one-factor models (standardized mediator and outcome scale items loading onto the same factor) and two-factor models (standardized mediator and outcome scale items loading onto separate factors) were evaluated in terms of factor loadings and model fit (i.e. Model Chi-Square; Comparative Fit Index, CFI; Root Mean Square Error of Approximation, RMSEA). Analyses were conducted in SPSS version 26 and $\mathrm{R}$ using the mitml (Grund et al. 2019), nlme (Pinheiro et al. 2020), eemeans (Lenthe et al. 2020) and laavan packages (Rosseel 2012).

\section{Results}

\section{Baseline Demographics and Clinical Characteristics}

From the total sample of 361 participants, median age was $29(\mathrm{IQR}=18), 70.6 \%(\mathrm{n}=258)$ of the sample was female and $84.2 \%(n=304)$ had white ethnicity. The majority of the sample was employed $(74.5 \%, n=269), 17.1 \%(n=61)$ had a comorbid long-term condition and $43.8 \%(n=158)$ was taking psychotropic medication. With regards to diagnoses as measured by the M.I.N.I.7.0.2., 80.3\% $(n=290)$ of the sample had at least one diagnosis of major depressive disorder $(52.4 \%, \mathrm{n}=189)$, anxiety disorder $(64 \%, \mathrm{n}=231)$ or comorbid diagnosis of depression and anxiety disorders $(36 \%, \mathrm{n}=130)$. Mean score for PHQ-9 and GAD were 14.3 $(\mathrm{SD}=5)$ and $12.6(\mathrm{SD}=4.5)$, respectively, indicating moderate levels of depression and anxiety. Mean scores at baseline for PBRS-A were 25.95 ( $\mathrm{SD}=5.2$ ), for the reappraisal subscale of the ERQ were $21.92(\mathrm{SD}=6.54)$ and for the suppression subscale of the ERQ were $16.03(\mathrm{SD}=4.9)$. No significant differences between conditions were found for any of the baseline demographic and clinical characteristics. Missing data at 8 weeks was $19.77 \%$ for the PRBS and $19.6 \%$ for the ERQ. Little's MCAR test suggested data to be missing at random for both, ERQ $\left(\chi^{2}(2)=4.2, p=.120\right)$ and PBRS-A $\left(\chi^{2}(1)=.914, p=.339\right)$.

\section{Change in Emotion Regulation Skills and Positive Beliefs About Rumination}

Random intercept linear mixed models with ERQ-A as the outcome variable indicated a statistically significant interaction effects of time-by-intervention-arm $(b=1.83, S E=0.82$, $95 \%$ CI $0.22-3.44, p=.026$ ). Bonferroni adjusted pairwise comparisons showed that participants in the interventionarm engaged in significantly more emotion regulation through cognitive reappraisal at 8 -weeks than at baseline (estimated mean difference 2.74, $\mathrm{SE}=0.48, \mathrm{t}(356)=5.75$, $\mathrm{p}<.0001$ ), while those in the waitlist control group did not (estimated mean difference $0.91, \mathrm{SE}=0.69, \mathrm{t}(356)=1.32$, $\mathrm{p}=1.0)$. The estimated between-group difference at 8 -weeks was $2.15(\mathrm{SE}=0.83, \mathrm{t}(356)=2.60, \mathrm{p}=.059)$. The corresponding models for ERQ-S and PBRS-A, revealed no statistically significant interaction effects of time-by-intervention-arm (ERQ-S: $b=-0.32, S E=0.54, C I-1.39$ to 0.74 , $p=.55$; PBRS-A: $b=0.89, S E=0.63, C I-0.34$ to 2.12 , $p=.16$ ), suggesting that iCBT did not lead to significant changes in these measures (Table 2).

\section{Mediation Effects of Emotion Regulation and Positive Beliefs About Rumination on Changes in Depression and Anxiety Outcomes}

Two parallel multiple mediation models were built (see Figs. 1, 2). Across both models only emotion regulation through cognitive reappraisal was found to be mediating the effects of iCBT on symptoms of depression and anxiety, fulfilling all required conditions for partial mediation to be concluded in line with Baron and Kenny (1986). In particular, the models showed that intervention-arm predicted change in ERQ-A scores (PHQ-9 model: $b=2.00$, $S E=0.81, p=0.014 ;$ GAD-7 model: $b=2.01, S E=0.81$,

Table 2 Estimated means for PBRS-A and ERQ subscales across groups and time-points

\begin{tabular}{clllll}
\hline Measure & \multicolumn{2}{l}{ Treatment group } & & \multicolumn{2}{l}{ Control group } \\
& Mean & SE & & Mean & SE \\
\hline Baseline & & & & \\
PBRS-A & 26 & 0.35 & & 25.8 & 0.49 \\
ERQ-S & 15.8 & 0.33 & 16.4 & 0.47 \\
ERQ-A & 22 & 0.44 & 21.7 & 0.64 \\
8-weeks & & & & \\
PBRS-A & 25.4 & 0.37 & 24.3 & 0.53 \\
ERQ-S & 14.9 & 0.35 & 15.8 & 0.50 \\
ERQ-A & 24.8 & 0.48 & 22.6 & 0.69 \\
\hline
\end{tabular}

$E R Q-A$ Emotion Regulation Questionnaire-Reappraisal subscale, $E R Q-S$ Emotion Regulation Questionnaire-Suppression subscale, $P B R S-A$ Positive Beliefs about Rumination Scale-adapted version, $S E$ standard error 
Fig. 1 Multiple mediation model of iCBT effects on depression outcomes (PHQ-9 model). Diagram depicting mediation model with regression coefficients and standard errors for each relationship estimated in the model. $* p<.05$, $* * p<.01$. ERQ $A$ - Emotion Regulation Questionnaire-Reappraisal subscale; $E R Q-S$ Emotion Regulation QuestionnaireSuppression subscale; $P B R S-A$ Positive Beliefs about Rumination Scale-adapted version
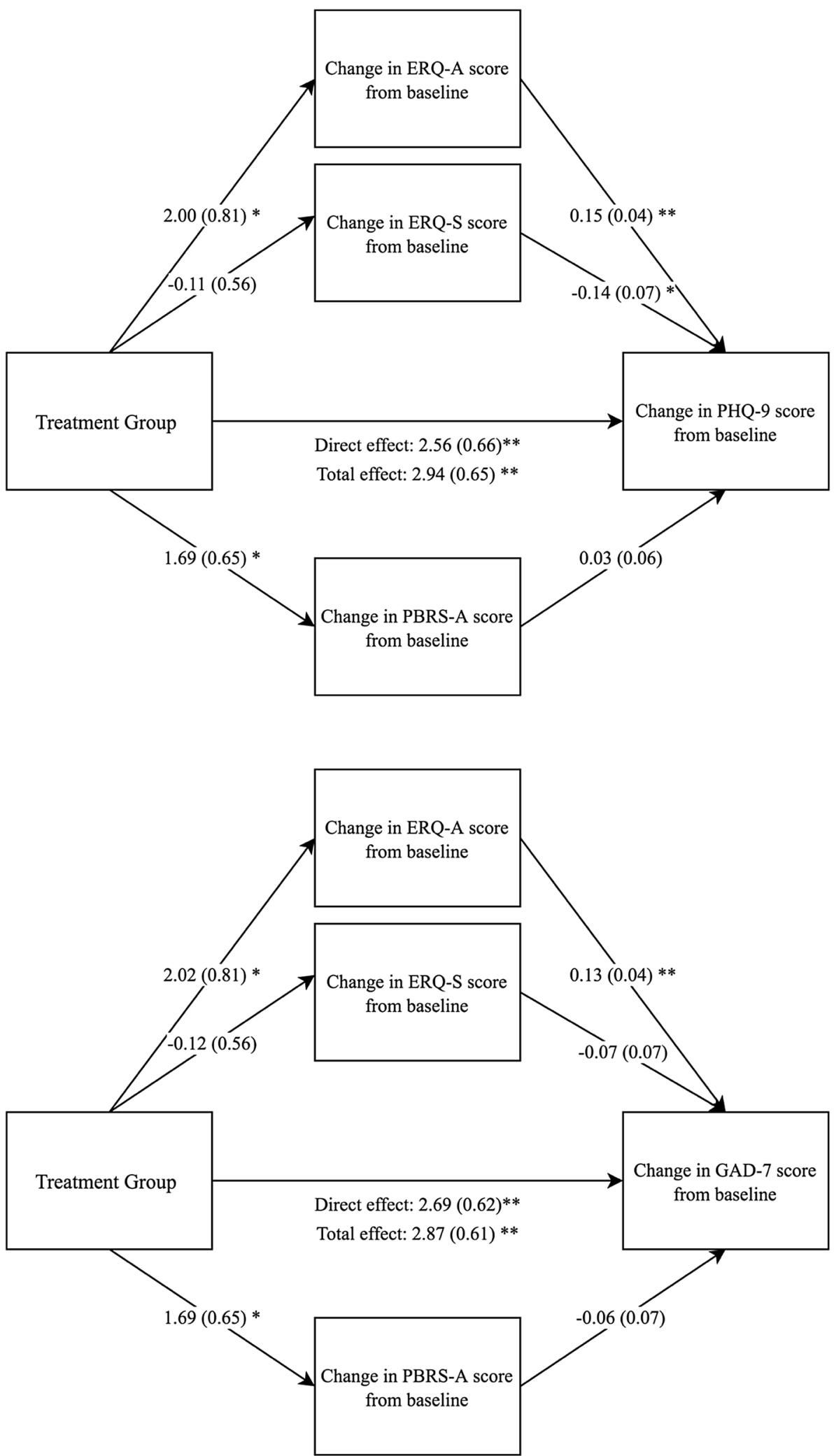

Fig. 2 Multiple mediation model of iCBT effects on anxiety outcomes (GAD-7 model). Diagram depicting mediation model with regression coefficients and standard errors for each relationship estimated in the model. $* \mathrm{p}<.05, * * \mathrm{p}<.01$. ERQ-A Emotion Regulation Questionnaire-Reappraisal subscale; ERQ-S Emotion Regulation Questionnaire-Suppression subscale; PBRS-A Positive Beliefs about Rumination Scaleadapted version $p=0.013)$, which in turn predicted change in PHQ-9 $(b=0.15, S E=0.04, p<.0001)$ and GAD-7 scores $(b=0.13$, $S E=0.04, p=0.002)$. The indirect effect was significant in the PHQ-9 model $(b=0.31, S E=0.15, p=0.043)$ and bordering significance in the GAD-7 model $(b=0.27, S E=0.14$, $p=0.057$ ). Post hoc confirmatory factor analyses supported 
the independence of constructs measured by the ERQ-A, PHQ-9 and GAD-7. Two-factor models in which individual ERQ-A, PHQ-9 and GAD-7 items loaded onto the factors of cognitive reappraisal, depression and anxiety, respectively, provided significantly better fit to the data than a one-factor solution (Chi-Squared Difference Test ERQ-A \& PHQ-9 models $\chi^{2}(1)=887.45, p<.0001$; ERQ-A \& GAD-7 models $\left.\chi^{2}(1)=852.62, p<.0001\right)$. Modification indices suggested improved model fit in relation to the inclusion of covariances between items of a scale but not in terms of factor loadings or covariances of items across different scales. All individual item factor loadings were significant (factor loadings ERQ-A 0.42-0.87; PHQ-9 0.40-75; GAD-7 0.35-0.86) and fit indices supported good model fit (ERQ-A \& PHQ-9 model: $\chi^{2}(79)=94.62, p=0.111 ; \mathrm{CFI}=0.991 ; \mathrm{RMSEA}=0.024$, $p=.998$; ERQ-A \& GAD-7 model: $\chi^{2}(58)=62.12$, $p=0.332$; CFI $=0.998$; RMSEA $=0.014, p=.999)$.

In terms of the ERQ-S, while reductions in emotion regulation via expressive suppression predicted depression symptom improvement (PHQ-9 model: $b=-0.14$, $S E=0.07, p=0.039$ ), no relationship between intervention-arm and change on the ERQ-S was found, rendering it insignificant as a mediator. In terms of the PBRS-A, both models suggested that waitlist-group assignment was related to greater reductions in positive beliefs about rumination (PHQ-9 model: $b=1.69, S E=0.65, p=0.01$; GAD-7 model: $b=1.69, S E=0.65, p=0.01)$. Changes in PBRS-A scores were unrelated to changes in anxiety and depression symptoms (PHQ-9 model: $b=1.69, S E=0.65, p=0.01$; GAD-7 model: $b=1.69, S E=0.65, p=0.01$ ), disqualifying it as a mediator. The direct effects of the intervention on clinical outcomes remained significant after accounting for all three mediators (PHQ-9 model: $b=2.56, S E=0.66, p<.0001$; GAD-7 model: $b=2.69, S E=0.62, p<.0001$ ), indicating that changes in ERQ-A only partially mediated treatment effects.

\section{Discussion}

This secondary analysis study explored the effect that iCBT interventions for depression and anxiety had on specific cognitive processes, such as metacognitive beliefs about rumination and emotion regulation skills, and preliminary mediation analyses were conducted to explore if these acted as potential mediators of the change observed in depression and anxiety symptoms at post-treatment (Richards et al. 2020). Overall, results showed that the intervention produced statistically significant improvements in cognitive reappraisal skills relevant for emotion regulation compared to the control group; however no between-group differences were observed for suppression of emotion expression and positive beliefs about rumination. Similarly, only reappraisal was found as a mediator of the change for both depression and anxiety, whereas suppression of emotion expression and positive beliefs about rumination were not found to be significant mediators on depression and anxiety.

The significant improvements observed in cognitive reappraisal skills are in line with previous studies for depression which found that individuals who went through iCBT improved emotion regulation skills via cognitive reappraisal after using the intervention (Forkmann et al. 2014; Ebert et al. 2013). Increases in reappraisal scores may be elevated in this iCBT intervention as this type of emotional regulation skill relates closely to the CBT concept of cognitive restructuring which is a cornerstone in the treatment process and is consistently worked on throughout the program (Aldao et al. 2014). Furthermore, the present study showed that reappraisal skills acted as a potential mediator for the changes observed in depression and anxiety symptoms, indicating that this is potentially a transdiagnostic shared mechanism that is successfully addressed by these interventions. A recent mediator analysis demonstrated that improvements to emotion regulation preceded and mediated reductions in anxiety symptoms (Asnaani et al. 2020), however, they did not discriminate between strategies of reappraisal or suppression, instead assessing broad difficulties in emotion regulation and awareness. It is important to note that given that the current study does not capture temporal associations between cognitive reappraisal and the primary outcomes, we cannot infer causality.

In regard to expressive suppression, our results showed that no changes were observed in emotional suppression and consequently it was not found to act as a potential mediator of change. The lack of effects for emotional suppression has also been found in other studies of face to face CBT (Forkmann et al. 2014). This indicates that while classic CBT incorporates cognitive reappraisal for disorders such as depression to challenge cognitive distortions and negative beliefs, emotional suppression and the extent to which it is used by individuals is not affected by CBT theories, since less focus is placed on behavioral emotion regulation (Forkmann et al. 2014). Long-term use of emotional suppression is negatively associated with positive affect and perpetuates low mood and anxiety (Ioannidis and Siegling 2015). The consistency in the findings reinforces the notion that in order to tackle expressive suppression, including strategies aimed at increasing emotional expression could be considered for inclusion in future internet-based CBT programs as part of an emotional regulation skills module (Berking et al. 2008).

The absence of effects on positive beliefs about rumination suggests that it is not associated with change in iCBT, since reductions in anxiety and depression symptoms occurred without co-occurring decrements or mediator effects of metacognitive beliefs. This is consistent with previous literature in depression where recovered individuals 
still showed elevated levels of positive metacognitive beliefs compared to depressed peers (Watkins and Moulds 2005). These findings contradict the results observed by Newby et al. (2014) who found that iCBT caused changes in positive metacognitive beliefs and these mediated the change in depression and anxiety symptoms. Of interest, the treatment components in that study differed in that they included specific components to target these beliefs (i.e. challenging positive beliefs about repetitive negative thinking) whereas these components were not present in the current intervention. Thus, more research is needed to determine how relevant metacognitive beliefs are for the treatment of depression and anxiety, especially considering that they are proxies for worry and rumination which have been found to be related to depression and anxiety (de Jong-Meyer et al. 2009; Freeston et al. 1994).

There are several strengths and limitations to this study. This is one of the largest controlled studies exploring specific mediators of change in iCBT interventions for depression and anxiety. Furthermore, the pragmatic nature of the trial, which was embedded around routine service procedures in IAPT increases the ecological validity of the findings. Regarding limitations, this sub study is exploratory in nature. One limitation is that PBRS-A and ERQ measures were collected in the same timeframe as primary outcomes. Thus, because atemporal mediation analyses do not allow us to attribute causality, we can only understand the mediation analyses as associations between the mediator variable and the outcomes rather than associations between causes and consequences. Confirmatory factor analyses were able to show the independence of outcome and mediator constructs; however, given that we could not assert whether changes in cognitive reappraisal occurred prior to changes in anxiety and depression our analyses were unable to determine whether cognitive reappraisal was in fact a mediator of outcomes or an outcome of iCBT in its own right. Future studies should measure outcome and mediator variables repeatedly throughout treatment to account for temporality and confirm that reappraisal is a mediator of the change. This could be done by modelling weekly symptom change with the mediator (i.e. cognitive reappraisal) as time-varying, lagged predictor in mixed models. Another limitation of this study is the heterogeneity in terms of depression and anxiety disorders included in the study. The fact that different conditions are combined in the analyses might have resulted in specific relationships between positive metacognitive beliefs (e.g. relationship with depression) or emotion regulation skills (e.g. expressive suppression in social anxiety) and specific disorders not being detected. Future studies including different disorders should attempt to include a balanced distribution of diagnoses to analyze the specific association between each presentation and the mediator variables. Another limitation is related to the use of the adapted version of the PBRS, which removed references to depression and rumination to account for broader signals of repetitive negative thinking for emotional disorders. In the current study, given the framing of the questions (i.e. 'I need to think about things to find answers to how I feel' instead of 'I need to ruminate about my problems to find answers to my depression) and the way CBT concepts are elaborated in the program, users might have been confused about the meaning of the statements. Future studies should consider adapting further the PBRS scale to account for the specificities of each disorder and the treatment to ensure a proper understanding. A similar effort was done by Wong and Moulds (2010) who further adapted the PBRS-A scale for individuals with social anxiety to cover the rumination about social events.

\section{Conclusion}

This study found that reappraisal skills may be a potential partial mediator of the effects of iCBT on depression and anxiety outcomes; but did not find similar effects for emotional suppression. These results are in line with findings on face to face CBT, suggesting that these mechanisms of change are operating similarly across both types of delivery. Regarding positive beliefs about rumination, our results did not show any impact of iCBT on these outcomes despite the intervention being successful at reducing depression and anxiety, calling into question the relative relevance of this factor in relation to the treatment effects. This study adds to the scarce literature on mediators of effects in iCBT from which we can imply mechanisms of change in this type of treatments; it thus contributes towards the understanding of how these interventions operate. It is important to acknowledge that this study only looked at three possible mediators and did not account for all possible mediators that may have mediated symptom improvement. Future work should consider the inclusion and examination of a broader set of possible mediators, congruent with intervention content, and explore any mechanisms that may be operating differently in iCBT compared to traditionally delivered CBT.

Acknowledgements We wish to thank the R\&D and clinical team members at Berkshire NHS Foundation Trust service for assisting trial execution: Gabriella Clark and Emma Cole, BSc. We thank the employees of our dedicated clinical and innovation team at SilverCloud for providing administrative support and assisting data collection and analysis. We also thank the many patients who volunteered their time and efforts to participate in our trial. Employees at SilverCloud Health and the University of Dublin, Trinity College managed the data collection. All authors approved the decision to submit for publication.

Author Contributions The trial was conceptualized and designed by DR, LT and DD. Data acquisition, analysis, and interpretation was led 
by DR, AE, NE, JP and DD. Drafting of the paper was conducted by AE, NE, RW. Critical revision of the paper for important intellectual content was provided by DR, AE, NE, JP, LT, with contributions from $\mathrm{DD}$, and CE. All the statistical analysis was handled by AE and NE. DR obtained the funding for the trial. Administrative, technical, and material support for the conduct of the trial was provided by DD, and CE. Trial supervision was provided by DR, AE. All authors reviewed and approved the final paper for submission.

Funding The study was funded by SilverCloud Health and Berkshire Healthcare Foundation Trust.

Data Availability The dataset and code used for analysis within the current study are available upon request by emailing the corresponding author.

\section{Compliance with Ethical Standards}

Conflict of Interest SilverCloud Health is a commercial organization that sells its digital programs to commissioners within the NHS who provide the service free to patients through the Improving Access to Psychological Therapies (IAPT) program. Authors Enrique, Eilert, Wogan, Early, Duffy, Palacios, and Richards are employees of SilverCloud Health, developers of computerized psychological interventions for depression, anxiety, stress, and comorbid long-term conditions. Timulak serves as a research consultant for SilverCloud Health.

Informed Consent Informed consent was obtained from all individual participants included in the study.

Human and Animal Rights No animal studies were carried out by the authors for this article.

Open Access This article is licensed under a Creative Commons Attribution 4.0 International License, which permits use, sharing, adaptation, distribution and reproduction in any medium or format, as long as you give appropriate credit to the original author(s) and the source, provide a link to the Creative Commons licence, and indicate if changes were made. The images or other third party material in this article are included in the article's Creative Commons licence, unless indicated otherwise in a credit line to the material. If material is not included in the article's Creative Commons licence and your intended use is not permitted by statutory regulation or exceeds the permitted use, you will need to obtain permission directly from the copyright holder. To view a copy of this licence, visit http://creativecommons.org/licenses/by/4.0/.

\section{References}

Aldao, A., Jazaieri, H., Goldin, P. R., \& Gross, J. J. (2014). Adaptive and maladaptive emotion regulation strategies: Interactive effects during CBT for social anxiety disorder. Journal of Anxiety Disorders, 28(4), 382-389. https://doi.org/10.1016/j.janxd is.2014.03.005.

Aldao, A., Nolen-Hoeksema, S., \& Schweizer, S. (2010). Emotionregulation strategies across psychopathology: A meta-analytic review. Clinical Psychology Review, 30(2), 217-237. https://doi. org/10.1016/j.cpr.2009.11.004.

Alonso, J., Liu, Z., Evans-Lacko, S., Sadikova, E., Sampson, N., Chatterji, S., et al. (2018). Treatment gap for anxiety disorders is global: Results of the World Mental Health Surveys in 21 countries. Depression and Anxiety, 35(3), 195-208. https://doi. org/10.1002/da.22711.

Andersson, G., \& Cuijpers, P. (2009). Internet-based and other computerized psychological treatments for adult depression: A metaanalysis. Cognitive Behaviour Therapy, 38(4), 196-205. https:// doi.org/10.1080/16506070903318960.

Andrews, G., Basu, A., Cuijpers, P., Craske, M. G., McEvoy, P., English, C. L., \& Newby, J. M. (2018). Computer therapy for the anxiety and depression disorders is effective, acceptable and practical health care: An updated meta-analysis. Journal of Anxiety Disorders, 55(4), 70-78. https://doi.org/10.1016/j.janxdis.2018.01.001.

Asnaani, A., Tyler, J., McCann, J., Brown, L., \& Zang, Y. (2020). Anxiety sensitivity and emotion regulation as mechanisms of successful CBT outcome for anxiety-related disorders in a naturalistic treatment setting. Journal of Affective Disorders, 267(4), 86-95. https://doi.org/10.1016/j.jad.2020.01.160.

Baron, R. M., \& Kenny, D. A. (1986). The moderator-mediator variable distinction in social psychological research: Conceptual, strategic, and statistical considerations. Journal of Personality and Social Psychology, 51(6), 1173-1182.

Bennett-Levy, J., Richards, D. A., Farrand, P., Bennett-Levy, J., Richards, D. A., \& Farrand, P. (2010). Low intensity CBT interventions: A revolution in mental health care. In Oxford guide to low intensity CBT interventions (pp. 3-18). https://doi.org/10.1093/ med:psych/9780199590117.003.0001.

Berking, M., Wupperman, P., Reichardt, A., Pejic, T., Dippel, A., \& Znoj, H. (2008). Emotion-regulation skills as a treatment target in psychotherapy. Behaviour Research and Therapy, 46(11), 1230-1237. https://doi.org/10.1016/j.brat.2008.08.005.

Brenes, G. A., Penninx, B. W. J. H., Judd, P. H., Rockwell, E., Sewell, D. D., \& Wetherell, J. L. (2008). Anxiety, depression and disability across the lifespan. Aging and Mental Health, 12(1), 158-163. https://doi.org/10.1080/13607860601124115.

Cook, L., Mostazir, M., \& Watkins, E. (2019). Reducing stress and preventing depression (RESPOND): Randomized controlled trial of web-based rumination-focused cognitive behavioral therapy for high-ruminating university students. Journal of Medical Internet Research, 21(5), 1-18. https://doi.org/10.2196/11349.

Cuijpers, P., Van Straten, A., Andersson, G., \& Van Oppen, P. (2008). Psychotherapy for depression in adults: A meta-analysis of comparative outcome studies. Journal of Consulting and Clinical Psychology, 76(6), 909-922. https://doi.org/10.1037/a0013075. supp.

de Jong-Meyer, R., Beck, B., \& Riede, K. (2009). Relationships between rumination, worry, intolerance of uncertainty and metacognitive beliefs. Personality and Individual Differences, 46(4), 547-551. https://doi.org/10.1016/j.paid.2008.12.010.

Dear, B. F., Staples, L. G., Terides, M. D., Karin, E., Zou, J., Johnston, L., et al. (2015). Transdiagnostic versus disorder-specific and clinician-guided versus self-guided internet-delivered treatment for generalized anxiety disorder and comorbid disorders: A randomized controlled trial. Journal of Anxiety Disorders, 36(12), 63-77. https://doi.org/10.1016/j.janxdis.2015.09.003.

Ebert, D., Tarnowski, T., Gollwitzer, M., Sieland, B., \& Berking, M. (2013). A transdiagnostic internet-based maintenance treatment enhances the stability of outcome after inpatient cognitive behavioral therapy: A randomized controlled trial. Psychotherapy and Psychosomatics, 82(4), 246-256. https://doi.org/10.1159/00034 5967.

Forand, N. R., Barnett, J. G., Strunk, D. R., Hindiyeh, M. U., Feinberg, J. E., \& Keefe, J. R. (2018). Efficacy of guided iCBT for depression and mediation of change by cognitive skill acquisition. Behavior Therapy, 49(2), 295-307. https://doi.org/10.1016/j. beth.2017.04.004.

Forkmann, T., Scherer, A., Pawelzik, M., Mainz, V., Drueke, B., Boecker, M., \& Gauggel, S. (2014). Does cognitive behavior 
therapy alter emotion regulation in inpatients with a depressive disorder? Psychology Research and Behavior Management, 7(5), 147-153. https://doi.org/10.2147/PRBM.S59421.

Freeston, M. H., Rhéaume, J., Letarte, H., Dugas, M. J., \& Ladouceur, R. (1994). Why do people worry? Personality and Individual Differences, 17(6), 791-802. https://doi.org/10.1016/01918869(94)90048-5.

Grek, A. (2007). Clinical management of suicidality in the elderly: An opportunity for involvement in the lives of older patients. Canadian Journal of Psychiatry, 52(6), 47.

Gross, J. J., \& John, O. P. (2003). Individual differences in two emotion regulation processes: Implications for affect, relationships, and well-being. Journal of Personality and Social Psychology, 85(2), 348-362. https://doi.org/10.1037/0022-3514.85.2.348.

Grund, S., Lüdtke, O., \& Robitzsch, A. (2016). Multiple imputation of multilevel missing data. SAGE Open, 6(4), 1-17. https://doi. org/10.1177/2158244016668220.

Grund, S., Robitzsch, A., \& Luedtke, O. (2019). mitml: Tools for multiple imputation in multilevel modeling. In $R$ package (pp. 1-50). https://cran.r-project.org/package $=$ mitml.

Holmes, E. A., Ghaderi, A., Harmer, C. J., Ramchandani, P. G., Cuijpers, P., Morrison, A. P., et al. (2018). The Lancet Psychiatry Commission on psychological treatments research in tomorrow's science. The Lancet Psychiatry, 5(3), 237-286. https://doi. org/10.1016/S2215-0366(17)30513-8.

Huque, M. H., Carlin, J. B., Simpson, J. A., \& Lee, K. J. (2018). A comparison of multiple imputation methods for missing data in longitudinal studies 01 Mathematical Sciences. BMC Medical Research Methodology, 18(1), 1-16. https://doi.org/10.1186/ s12874-018-0615-6.

Ioannidis, C. A., \& Siegling, A. B. (2015). Criterion and incremental validity of the emotion regulation questionnaire. Frontiers in Psychology, 6, 247. https://doi.org/10.3389/fpsyg.2015.00247.

James, S. L., Abate, D., Abate, K. H., Abay, S. M., Abbafati, C., Abbasi, N., et al. (2018). Global, regional, and national incidence, prevalence, and years lived with disability for 354 Diseases and Injuries for 195 countries and territories, 19902017: A systematic analysis for the Global Burden of Disease Study 2017. The Lancet, 392(10159), 1789-1858. https://doi. org/10.1016/S0140-6736(18)32279-7.

Kazdin, A. E. (2007). Mediators and mechanisms of change in psychotherapy research. Annual Review of Clinical Psychology, 3(1), 1-27. https://doi.org/10.1146/annurev.clinpsy.3.02280 6.091432 .

Kertz, S. J., Koran, J., Stevens, K. T., \& Björgvinsson, T. (2015). Repetitive negative thinking predicts depression and anxiety symptom improvement during brief cognitive behavioral therapy. Behaviour Research and Therapy, 68(5), 54-63. https://doi.org/10.1016/j. brat.2015.03.006.

Kessler, R. C., Merikangas, K. R., \& Wang, P. S. (2007). Prevalence, comorbidity, and service utilization for mood disorders in the United States at the beginning of the twenty-first century. Annual Review of Clinical Psychology, 3(1), 137-158. https://doi. org/10.1146/annurev.clinpsy.3.022806.091444.

Kroenke, K., Spitzer, R. L., \& Williams, J. B. W. (2001). The PHQ-9: Validity of a brief depression severity measure. Journal of General Internal Medicine, 16(9), 606-613. https://doi.org/10.104 6/j.1525-1497.2001.016009606.x.

Lenthe, R., Buerkner, P., Herve, M., Love, J., Riebl, H., \& Singmann, H. (2020). Package "emmeans" type package title estimated marginal means, aka least-squares means. $R$ Package. https://doi. org/10.1080/00031305.1980.10483031.

McEvoy, P. M., Erceg-Hurn, D. M., Anderson, R. A., Campbell, B. N. C., \& Nathan, P. R. (2015). Mechanisms of change during group metacognitive therapy for repetitive negative thinking in primary and non-primary generalized anxiety disorder. Journal of Anxiety Disorders, 35, 19-26. https://doi.org/10.1016/j.janxd is.2015.07.003.

Mogoașe, C., Cobeanu, O., David, O., Giosan, C., \& Szentagotai, A. (2017). Internet-based psychotherapy for adult depression: What about the mechanisms of change? Journal of Clinical Psychology, 73(1), 5-64. https://doi.org/10.1002/jclp.22326.

Newby, J. M., Williams, A. D., \& Andrews, G. (2014). Reductions in negative repetitive thinking and metacognitive beliefs during transdiagnostic internet cognitive behavioural therapy (iCBT) for mixed anxiety and depression. Behaviour Research and Therapy, 59(8), 52-60. https://doi.org/10.1016/j.brat.2014.05.009.

Newman, M. G., Zuellig, A. R., Kachin, K. E., Constantino, M. J., Przeworski, A., Erickson, T., \& Cashman-McGrath, L. (2002). Preliminary reliability and validity of the generalized anxiety disorder questionnaire-IV: A revised self-report diagnostic measure of generalized anxiety disorder. Behavior Therapy, 33(2), 215233. https://doi.org/10.1016/S0005-7894(02)80026-0.

NICE. (2009). Depression in Adults (update) Depression: The treatment and management of depression in adults. In National Clinical Practice Guideline Number X. www.nice.org.uk/guidance/ cg90.

NICE. (2011). Generalised anxiety disorder and panic disorder (with or without agoraphobia) in adults: Management in primary, secondary and community care (partial update). In National Clinical Guideline Number 113 (Issue 113). www.nice.org.uk/guidance/ TA97.

Papageorgiou, C., \& Wells, A. (2001). Positive beliefs about depressive rumination: Development and preliminary validation of a self-report scale. Behavior Therapy, 32(1), 13-26. https://doi. org/10.1016/S0005-7894(01)80041-1.

Pinheiro, J., Bates, D., DebRoy, S., Sarkar, D., \& Team, R. C. (2020). nlme: Linear and nonlinear mixed effects models description. In $R$ package: Vol. version 3 (pp. 1-149). Comprehensive R Archive Network (CRAN). https://cran.r-project.org/package=nlme.

Richards, D., Duffy, D., Blackburn, B., Earley, C., Enrique, A., Palacios, J., et al. (2018). Digital IAPT: The effectiveness \& costeffectiveness of internet-delivered interventions for depression and anxiety disorders in the Improving Access to Psychological Therapies programme: Study protocol for a randomised control trial. BMC Psychiatry, 18(1), 59. https://doi.org/10.1186/s1288 8-018-1639-5.

Richards, D., Enrique, A., Eilert, N., Franklin, M., Palacios, J., Duffy, D., et al. (2020). A pragmatic randomized waitlist-controlled effectiveness and cost-effectiveness trial of digital interventions for depression and anxiety. Npj Digital Medicine, 3(1), 1-10. https ://doi.org/10.1038/s41746-020-0293-8.

Richards, D., Timulak, L., O’Brien, E., Hayes, C., Vigano, N., Sharry, J., et al. (2015). A randomized controlled trial of an internetdelivered treatment: Its potential as a low-intensity community intervention for adults with symptoms of depression. Behaviour Research and Therapy, 75(12), 20-31. https://doi.org/10.1016/j. brat.2015.10.005.

Richards, D., Timulak, L., Rashleigh, C., McLoughlin, O., Colla, A., Joyce, C., et al. (2016). Effectiveness of an internet-delivered intervention for generalized anxiety disorder in routine care: A randomised controlled trial in a student population. Internet Interventions, 6(11), 80-88. https://doi.org/10.1016/j.inven t.2016.10.003.

Rodebaugh, T. L., Holaway, R. M., \& Heimberg, R. G. (2008). The factor structure and dimensional scoring of the generalized anxiety disorder questionnaire for DSM-IV. Assessment, 15(3), 343-350. https://doi.org/10.1177/1073191107312547.

Rosseel, Y. (2012). Lavaan: An R package for structural equation modeling. Journal of Statistical Software, 48(1), 1-36. https:// doi.org/10.18637/jss.v048.i02. 
Salomonsson, S., Santoft, F., Lindsäter, E., Ejeby, K., Ingvar, M., Öst, L. G., et al. (2019). Predictors of outcome in guided selfhelp cognitive behavioural therapy for common mental disorders in primary care. Cognitive Behaviour Therapy. https://doi. org/10.1080/16506073.2019.1669701.

Sheehan, D. V., Lecrubier, Y., Sheehan, K. H., Janavs, J., Weiller, E., Keskiner, A., et al. (1997). The validity of the Mini International Neuropsychiatric Interview (MINI) according to the SCID-P and its reliability. European Psychiatry, 12(5), 232-241. https://doi. org/10.1016/S0924-9338(97)83297-X.

Sloan, E., Hall, K., Moulding, R., Bryce, S., Mildred, H., \& Staiger, P. K. (2017). Emotion regulation as a transdiagnostic treatment construct across anxiety, depression, substance, eating and borderline personality disorders: A systematic review. Clinical Psychology Review, 57(11), 141-163. https://doi.org/10.1016/j. cpr.2017.09.002.

Spitzer, R. L., Kroenke, K., Williams, J. B. W., \& Löwe, B. (2006). A brief measure for assessing generalized anxiety disorder: The GAD-7. Archives of Internal Medicine, 166(10), 1092-1097. https ://doi.org/10.1001/archinte.166.10.1092.

Thornicroft, G., Chatterji, S., Evans-Lacko, S., Gruber, M., Sampson, N., Aguilar-Gaxiola, S., et al. (2017). Undertreatment of people with major depressive disorder in 21 countries. British Journal of Psychiatry, 210(2), 119-124. https://doi.org/10.1192/bjp. bp.116.188078.

Topper, M., Emmelkamp, P. M. G., Watkins, E., \& Ehring, T. (2017). Prevention of anxiety disorders and depression by targeting excessive worry and rumination in adolescents and young adults: A randomized controlled trial. Behaviour Research and Therapy, 90(3), 123-136. https://doi.org/10.1016/j.brat.2016.12.015.

Watkins, E., \& Moulds, M. (2005). Positive beliefs about rumination in depression-A replication and extension. Personality and Individual Differences, 39(1), 73-82. https://doi.org/10.1016/j. paid.2004.12.006.

Wong, Q. J. J., \& Moulds, M. L. (2010). Do socially anxious individuals hold positive metacognitive beliefs about rumination? Behaviour Change, 27(2), 69-83. https://doi.org/10.1375/bech.27.2.69.

Wright, J. H., Owen, J. J., Richards, D., Eells, T. D., Richardson, T., Brown, G. K., et al. (2019). Computer-assisted cognitive-behavior therapy for depression. The Journal of Clinical Psychiatry, 80(2). https://doi.org/10.4088/JCP.18r12188

Wu, Z., \& Fang, Y. (2014). Comorbidity of depressive and anxiety disorders: Challenges in diagnosis and assessment. Shanghai Archives of Psychiatry, 26(4), 227-231. https://doi.org/10.3969/j. issn.1002-0829.2014.04.006.

Yuan, K.-H., \& Bentler, P. M. (2000). 5. Three likelihood-based methods for mean and covariance structure analysis with nonnormal missing data. Sociological Methodology, 30(1), 165-200. https:// doi.org/10.1111/0081-1750.00078.

Publisher's Note Springer Nature remains neutral with regard to jurisdictional claims in published maps and institutional affiliations. 